\title{
Ag nanoparticle-decorated single wall carbon nanotube films for photovoltaic applications
}

\author{
Mokhtar Anouar ${ }^{1} \cdot$ Rhanem Jbilat $^{1} \cdot$ Vincent Le Borgne $^{1}$ • \\ Dongling $\mathrm{Ma}^{1} \cdot$ My Ali EI Khakani ${ }^{1}$
}

Received: 16 July 2015/ Accepted: 19 November 2015/Published online: 9 January 2016

(C) The Author(s) 2016. This article is published with open access at Springerlink.com

\begin{abstract}
We report on the use of pulsed laser deposition to decorate single wall carbon nanotube (SWCNTs) films with Ag nanoparticles (NPs) in hybrid SWCNTs/n-Si photovoltaic (PV) devices. PV devices are built by coating n-Si surfaces with a controlled density of a SWCNTs' suspension via an air-brush method. By adjusting the number of laser pulses $\left(N_{\mathrm{Lp}}\right)$ from the $\mathrm{KrF}$ laser, we are able to control the size and density of the Ag NPs covering the SWCNTs films. Through adjustment of $N_{\mathrm{Lp}}$, we are able to improve the power conversion efficiency of the SWCNTs/n-Si devices from 3.5 to over $6 \%$ at $N_{\mathrm{Lp}}=1250$ and the corresponding fill factor (FF) from 35 to $60 \%$. This increase is shown to be correlated with Raman, electrical and optical properties of the Ag NPs-coated SWCNTs films. UV-Vis spectroscopy measurements show the presence of optical scattering that is directly attributed to the presence of plasmon in the range of 450-600 nm and internal quantum efficiency measurement shows significant improvement over this range. In addition to this direct increase of the generate photocurrent, the overall Ag NPs film resistance is sufficiently lowered to ensure higher FF and thus a higher PCE. Beyond the optimal value of $N_{\text {Lp }}>1250$, we show that the decreasing PCE is caused by low optical transmission of the Ag NPs films and poorer rectification.
\end{abstract}

My Ali El Khakani

elkhakani@emt.inrs.ca

Vincent Le Borgne

leborgne@emt.inrs.ca

1 Institut National de la Recherche Scientifique, 1650 Lionel Boulet, Varennes, Quebec J3X 1S2, Canada
Keywords Silver nanoparticles - Single wall carbon nanotubes - Photovoltaic - Solar cells · Pulsed laser deposition

\section{Introduction}

Nanomaterial-based photovoltaic (PV) devices are attracting much attention as promising candidates for the design of third-generation solar cells. Among nanomaterials, single wall carbon nanotubes (SWCNTs) have shown to be a prime candidate for third-generation solar cells and an important topic of research. The unique qualities of SWNCTs, such as one-dimensional nanoscale structure, high aspect ratios, large specific surface area, high charge mobility [1], and excellent optical and electronic properties [2] have led to sustained and ever increasing research efforts over the past few years. SWCNTs have been integrated in a variety of photovoltaic devices including silicon-based [3], polymer-based [4] and dye sensitized solar cells (DSSC) [5], either as a photogenerating medium, as transparent electrodes or as scaffolding for other photoactive materials such as $\mathrm{TiO}_{2}$. Recently, SWCNTs/n-Si PV devices have evolved into their own field of research at the intersection of well-established silicon-based technology and cutting-edge nanomaterial science. These devices typically rely on nanoheterojunctions of n-type silicon and p-type SWCNTs films [6-11]. It has been reported that the transparency and electrical conductivity of the SWCNTs films play a direct role in the performance of such PV devices [12,13]. A balance has to be struck between the transparency of the SWCNTs film, to take full advantage of the silicon substrate, and conductivity of the SWCNTs to facilitate charge extraction. Therefore, most of the current research efforts on such SWCNTs/n-Si PV devices focus 
on the optimization of the transparency and the conductivity of the SWCNTs films to maximize the performance of these PV devices. We have shown in previous work that these two parameters can be directly altered through film fabrication procedures and combined into a figure of merit that correlates with PV properties [3]. In addition to the optimization of the SWCNTs' films via fabrication procedures, chemical doping, often through the addition of nitric acid [7, 9, 14], has been widely used as a method to increase conductivity without significantly altering transparency. Doping can achieve other desirable results: though SWCNTs are naturally p-doped [15], the addition of solutions such as $\mathrm{SOCl}_{2}$ [16] or $\mathrm{H}_{2} \mathrm{O}_{2}$ [17] to the SWCNTs leads to increased p-doping, which in turn improves the efficiency of the SWCNTs/n-Si junction. Improvement of the performances of the SWCNTs/n-Si devices has also been achieved by methods that rely on alterations achieved through physical means such as light trapping and plasmon enhancement. Indeed, recent work has shown that $\mathrm{TiO}_{2}$ films deposited by spin-coating and used an anti-reflection coating significantly increased the light absorption of the SWCNTs/n-Si devices [14]. The addition of silver nanowires has also been proposed to increase the effective conductivity of the SWCNTs films without significantly reducing optical transparency [18]. Similarly, recent work has introduced the idea of adding metal nanoparticles (NPs) to SWCNTs/n-Si. For instance, Ag NPs have been proposed as additional light scattering sites, thus increasing the light effectively available for photogeneration. Decoration of a solar cell with Ag NPs has been successfully used in thin film silicon solar cells $[19,20]$, showing a proof of concept in a well-understood type of solar cell and that this is a viable route to performance enhancement. The use of Ag NPs in SWCNTs/PV devices has also been investigated: while improvement has been reported, the maximal reported PCE value of only $1.32 \%$ [21] is low and likely could be improved upon.

In this work, we demonstrate the potential of pulsed laser deposition (PLD) to decorate SWCNTs films with $\mathrm{Ag}$ NPs. PLD is a synthesis method known to produce clean and uniform NPs over a surface, without the risk of chemical contamination that arises from chemical methods. It has been employed to decorate CNTs in several applications, including field effect emission [22] and optoelectronics [23, 24]. We show that the PLD method yields clean nanohybrids (NHs) and that the SWCNTs films are left structurally intact after the deposition process. The presence of the Ag surface plasmon in Raman spectroscopy and UV-Vis optical transmission properties is shown directly through Raman and optical transmission spectroscopy. Finally, the Ag NPs/SWCNTs NHs synthesized directly on $\mathrm{n}-\mathrm{Si}$ substrates were used to fabricate PV devices. The number of laser pulses $\left(N_{\mathrm{Lp}}\right)$ used during
PLD allows us to exert direct control over the size distribution and density of the Ag NPs within the NHs. We thus show that the PCE can be optimized by varying the

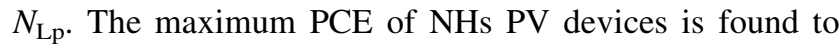
be almost two times that of devices built from pristine SWCNTs. This increase arises from improvements in the fill factor (FF) as well as from plasmon-induced effects. We also show that there appears to be a critical density of NPs that leads to a very strong increase of the PCE properties. This is attributed to the presence of a percolated continuous network of large $\mathrm{Ag}$ particles that inhibits the performance-enhancing quantum properties of $\mathrm{Ag}$ NPs.

\section{Experimental}

\section{Preparation of the SWCNTs devices}

The as-received SWCNTs (Carbon Solution) were suspended in di-methyl-formamide (DMF) at a concentration of $0.1 \mathrm{mg} / \mathrm{ml}$ with $30 \mathrm{~min}$ sonication in an ultrasonic bath. SWCNTs films were obtained by spray-coating $1 \times 1 \mathrm{~cm}$ $\mathrm{n}-\mathrm{Si}$ pieces. To ensure direct contact between SWCNTs and $\mathrm{n}-\mathrm{Si}$, the substrate is treated with dilute $\mathrm{HF}$ to remove native oxide immediately before SWCNTs deposition. SWCNTs films were optimized following a previously published procedure that relies on the optimization of a figure of merit (FoM $=T^{10} / R_{\mathrm{S}}$ ) [12] where $T$ is the optical transmission of the films at $550 \mathrm{~nm}$ and $R_{\mathrm{s}}$ is the sheet resistance. Typical values of the FoM were of $10^{-3} \Omega^{-1}$.

SWCNTs films were exposed for $2 \mathrm{~s}$ to nitric acid vapors to dope them and increase the PV response. After drying the deposited nitric acid, the samples are inserted in a PLD chamber to be decorated with Ag NPs. In this process, a $\mathrm{KrF}$ laser beam (248 nm, $20 \mathrm{~ns}, 165 \mathrm{~mJ} /$ pulse) is focalized onto an Ag target placed in a chamber with a 300 mTorr He background pressure. Ag NPs are deposited directly onto a variety of substrates including the SWCNTs/n-Si structures as well as bare Si and bare quartz substrates for parallel characterizations. The SWCNTs/n-Si structures were systematically decorated with Ag-NPs by varying the number of laser pulses $\left(N_{\mathrm{Lp}}\right)$.

\section{Materials' characterizations}

The optical transmission and reflection spectra of Ag NPs, SWCNTs films and Ag NPs-decorated SWCNTs films were obtained using a Varian Cary 5000. NPs sizes and SWNCTs films uniformity were characterized through SEM images (JEOL-6900). Finally, the Raman spectra were measured with a $514 \mathrm{Ar}^{+}$laser in a RM3300 Renishaw system. 


\section{Device characterization}

The external quantum efficiency (EQE) spectra of SWCNTs/n-Si devices were obtained using a lock-in amplifier (Ametek). Devices were illuminated by monochromatic light from $150 \mathrm{~W}$ Xe lamp chopped at $6 \mathrm{~Hz}$. Lamp power was systematically measured with a calibrated Si-diode (Newport). The EQE was obtained as follows: $\mathrm{EQE}=100 \times h c \times I_{\mathrm{ph}} / \lambda P$ where $P$ is the incident light power at a given wavelength $(\lambda), c$ is the speed of the light, $h$ is Planck constant and $I_{\mathrm{ph}}$ is the photocurrent generated by the device. Internal quantum efficiency was obtained by the following equation: $\mathrm{IQE}=\mathrm{EQE} /$ $(1-T)$ where $T$ is the optical transmission of either the $\mathrm{Ag}$ NPs/SWCNTs NHs or the pristine SWCNTs films.

$J-V$ measurements under solar radiation (Oriel AM 1.5 solar simulator) were performed with an Agilent 6900. All $J-V$ measurements were performed through a circular aperture of $2 \mathrm{~mm}$ diameter.

\section{Results}

\section{Materials' characterization}

Ag NPs are commonly used as an efficient material for surface-enhanced Raman spectroscopy (SERS). [25] Raman spectroscopy is thus a powerful first-line characterization tool for Ag-decorated SWCNTs and can easily detect any Ag-induced alterations. Figure 1 shows Raman spectra for selected values of $N_{\mathrm{Lp}}$, including pristine SWCNTs (bottom curve) up to $N_{\mathrm{Lp}}=2000$ (top curve). All spectra are measured under identical conditions, which allow for meaningful comparison of absolute Raman intensity. At $N_{\mathrm{Lp}}=0$ (i.e., pristine SWCNTs), the Raman spectrum is shown to have an intense $G$ band with respect to the D band, indicating the high quality of the SWCNTs [26]. The G-band line shape indicates that semiconductor SWCNTs are preferentially excited by the $\mathrm{Ar}^{+}$laser [27]. The RBM peak position (around $170 \mathrm{~cm}^{-1}$ ) arises from SWCNTs of $1.5 \mathrm{~nm}$ diameter [28]. There is a slight broadening of the RBM peak as the value of $N_{\mathrm{Lp}}$ is increased that is attributed to pressure the large amount of silver exerts on the SWCNTs wall. The RBM peak disappears entirely at $N_{\mathrm{Lp}}>1250$, indicating that beyond this threshold, the SWCNTs films' electronic properties could be drastically modified [29]. Additionally, the G band broadens and its two components overlap more. Despite these electronic changes, the $\mathrm{D}$ band, which is proportional to the density of defects, varies very little, indicating that the decoration of SWCNTs with Ag NPs does not induce defects. As the value of $N_{\mathrm{Lp}}$ is varied between 0 and 2000, intensity of the Raman spectra increases by a factor of 5 at
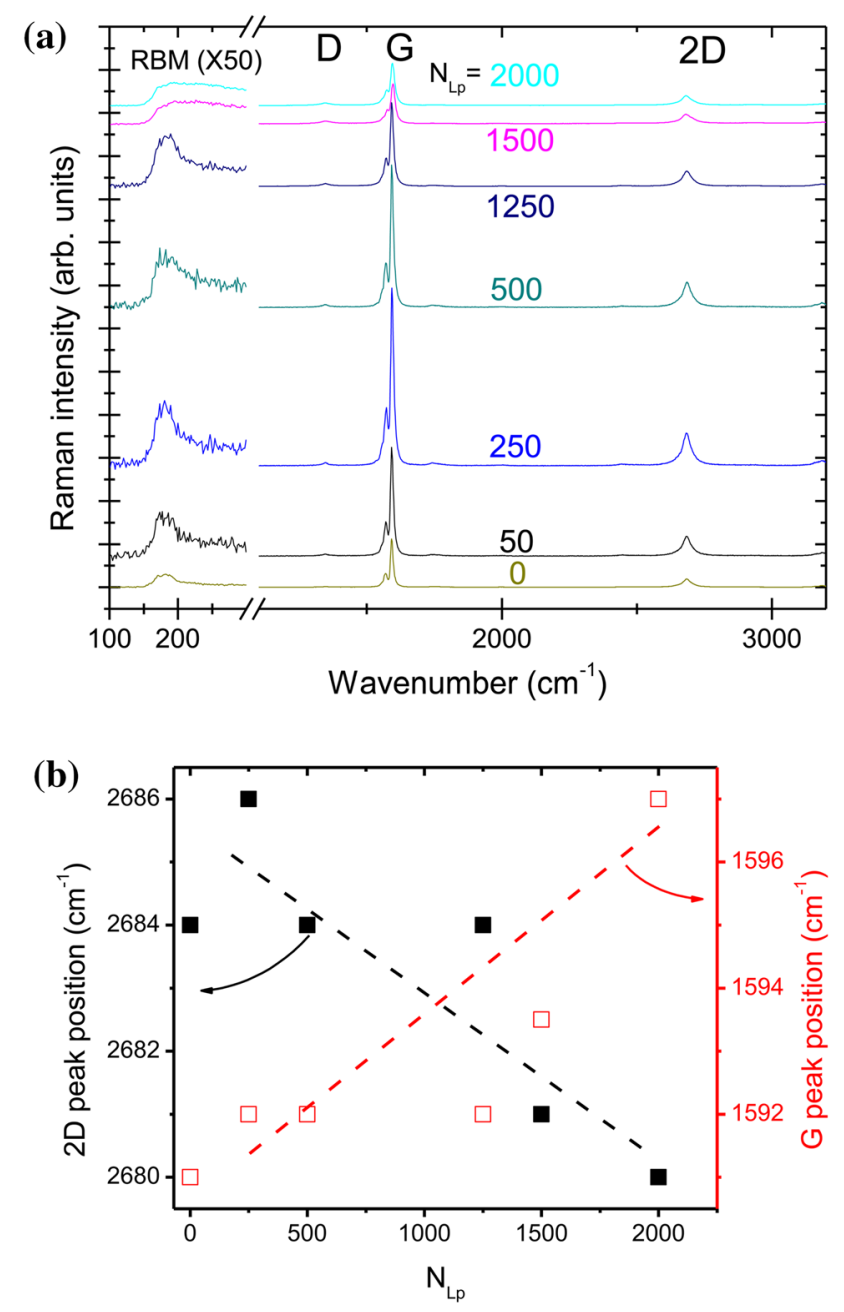

Fig. 1 a Comparison of the Raman spectra of SWCNTs films decorated with Ag NPs for selected values of $N_{\mathrm{Lp}}$. b Positions of the $2 \mathrm{D}$ and $\mathrm{G}$ peaks as a function of $N_{\mathrm{Lp}}$

$N_{\mathrm{Lp}}=250$ with respect to pristine SWCNTs. This increase is attributed to SERS induced by the presence of Ag NPs [30]. Starting at $N_{\mathrm{Lp}}=500$, the absolute Raman intensity decreases, and all signal enhancement has disappeared at $N_{\mathrm{Lp}}=1500$. Higher values of $N_{\mathrm{Lp}}$ would presumably yield larger NPs [24], which would have a gradually less pronounced SERS as a function of $N_{\mathrm{Lp}}$ until the Ag film fully percolates. Thus, when $N_{\mathrm{Lp}}$ goes beyond a critical threshold, the Ag NPs film behaves more as continuous films rather than as a collection of NPs, thus negating the effects of the NPs' SERS.

On top of SERS altering the Raman intensity, dopinglike behavior induced by the presence of Ag NPs can be observed directly through Raman spectroscopy. Indeed, starting at $N_{\mathrm{Lp}}=1500$, a significant shift of the $2 \mathrm{D}$ and G bands was observed [31-34]. The 2D band is shifted to lower frequency by $6 \mathrm{~cm}^{-1}$ (Fig. $1 \mathrm{~b}$ and the $\mathrm{G}$ band is shifted to higher frequency by about $7 \mathrm{~cm}^{-1}$, both typically 
signs of n-type doping [35]. Though there are no chemical bonds between $\mathrm{C}$ and $\mathrm{Ag}$, the physical proximity of the $\mathrm{Ag}$ NPs can induce local effects similar to charge transfer [34] and, in turn, n-type doping by making the $\mathrm{Ag}$ electrons available to the SWCNTs. To summarize, Raman spectroscopy shows that the SWCNTs/Ag NPs NHs are successfully synthesized through the increased intensity of the Raman signal, and that they significantly alter the electronic properties of the SWCNTs at high values of $N_{\mathrm{Lp}}$.

$\mathrm{Ag} \mathrm{NHs}$ were further investigated through SEM to assess the deposition efficiency of the PLD method as well and NPs size distribution. Figure 2 compares SEM images of NHs for several values of $N_{\mathrm{Lp}}$. At $N_{\mathrm{Lp}}=0$ (Fig. 2a), pristine SWCNTs are seen to form a continuous film of interwoven bundles that offer a very large effective area onto which the NPs can be deposited. It can also be seen that the SWCNTs are clean and devoid of contamination from amorphous carbon or other undesirable carbon species, which is in line with the low intensity D band of the Raman spectra. In Fig. 2b, the SEM image for Ag NPs deposited with $N_{\mathrm{Lp}}=500$ shows sparse and rather small diameters of NPs on the SWCNTs. As $N_{\mathrm{Lp}}$ is increased, the NPs grow in size and density until the SWCNTs are fully covered (Fig. 2d), corroborating the Raman spectroscopy results shown in Fig. 1. At $N_{\mathrm{Lp}}>1250$, the Ag NPs appear to form a continuous film over the SWCNTs bundles, mitigating any SERS and effectively acting as a single $\mathrm{Ag}$ layer. NPs size and density have been estimated through SEM images. Indeed, in Fig. 2e, the typical NPs size distribution is shown for three values of $N_{\mathrm{Lp}}\left(N_{\mathrm{Lp}}=500\right.$, 1250 and 2000) (e). The median size for $N_{\mathrm{Lp}}=500$ is found to be about $20 \mathrm{~nm}$ and it is pushed up to $40 \mathrm{~nm}$ at
Fig. 2 SEM images of SWCNTs films decorated with $\mathrm{Ag}$ NPs for selected values of $N_{\mathrm{Lp}}$ a $N_{\mathrm{Lp}}=0, \mathbf{b} N_{\mathrm{Lp}}=500$, c $N_{\mathrm{Lp}}=1250, \mathbf{d} N_{\mathrm{Lp}}=2000$, e diameter distributions of the Ag NPs
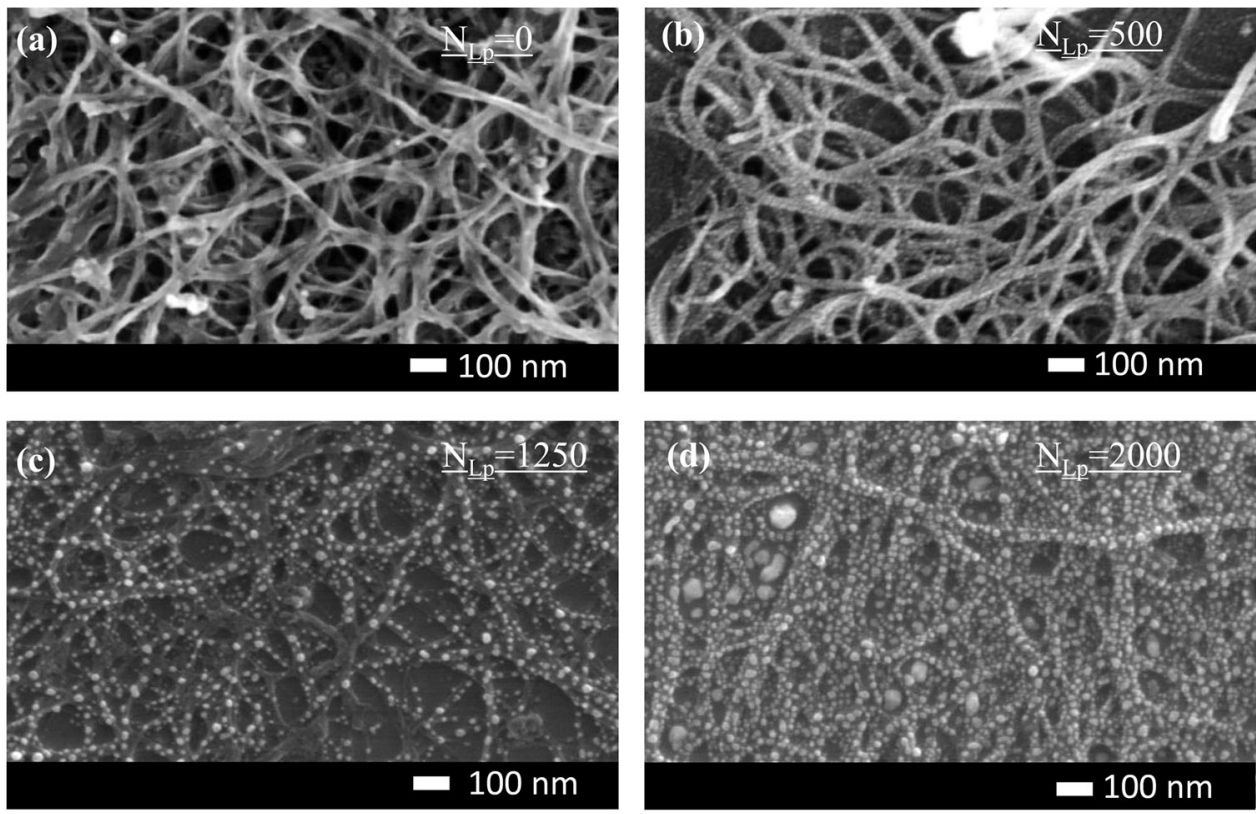

(e)

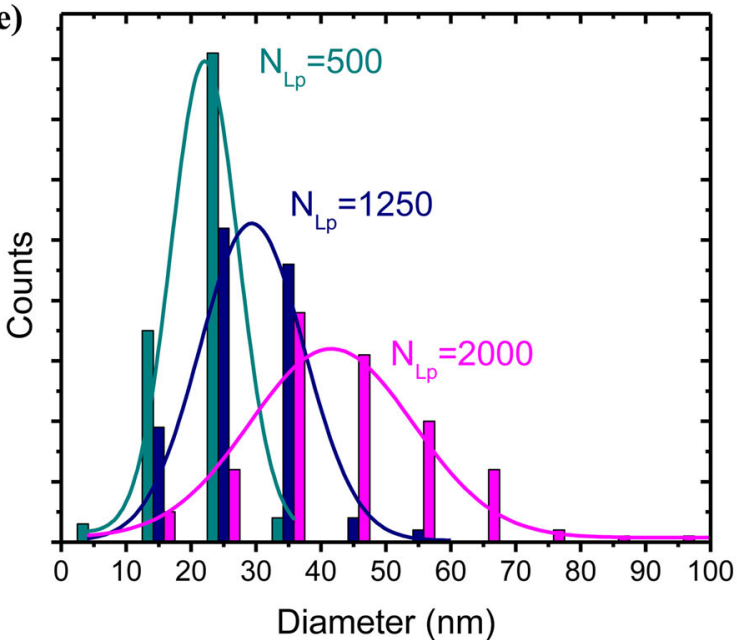


$N_{\text {Lp }}=2000$. This shift is accompanied by an increase in the spread in the NPs due to NP coalescence at high values of $N_{\mathrm{Lp}}$ (i.e., two initially distinct NPs that grow sufficiently to be conjoined), leading to particles with sizes ranging between 10 and $90 \mathrm{~nm}$ at $N_{\mathrm{Lp}}=2000$. Overall, SEM images show that both the median particle size and density are correlated with $N_{\mathrm{Lp}}$ and that the Ag NPs form continuous films at high values of $N_{\mathrm{Lp}}$.

UV-Vis spectroscopy measurements can provide additional information on the presence of plasmon resonance in $\mathrm{Ag}$ NPs. Figure 3a directly compares the UV-Vis transmission spectra of Ag NPs deposited on quartz under the same conditions as that of NPs deposited on SWCNTs films. $N_{\text {Lp }}$ as low as 50 generates NPs that have a distinct plasmon peak. At low values of $N_{\mathrm{Lp}}$, the plasmon peak is well defined with little to no loss in the infrared range. Conversely, as the number of laser pulses is increased, the plasmon peak broadens and red-shifts until the plasmon peak entirely disappears for $N_{\mathrm{Lp}}=2000$. Over this range of $N_{\mathrm{Lp}}$, the position of the dispersion peak shifts
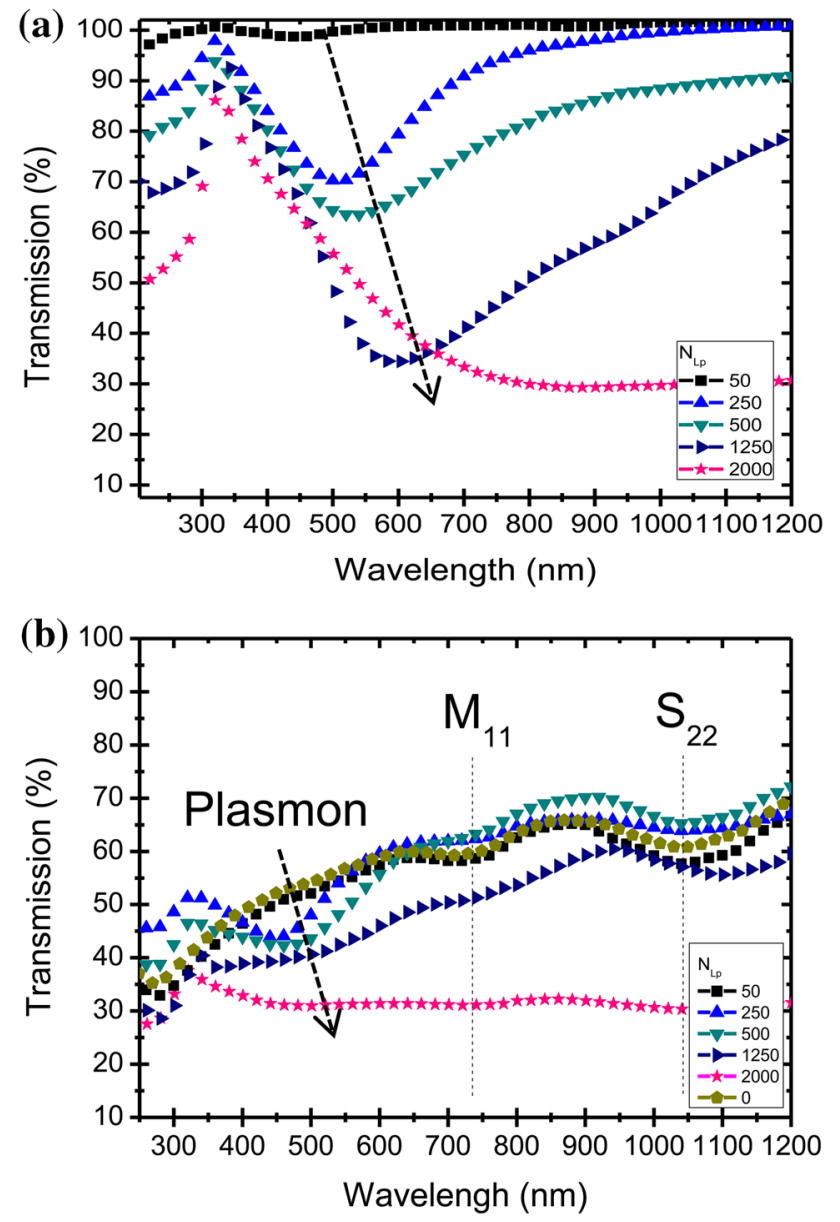

Fig. 3 a UV-Vis transmission spectra of Ag NPs deposited on quartz. b UV-Vis transmission spectra of SWCNTs films decorated with Ag NPs significantly, going from as low as $400 \mathrm{~nm}$ for $N_{\mathrm{Lp}}=50$ to almost $600 \mathrm{~nm}$ for $N_{\mathrm{Lp}}=1250$. The same measurements were also performed on SWCNTs/Ag-NPs NHs deposited on quartz. Figure $3 \mathrm{~b}$ shows the optical transmission spectra for NHs for selected values of $\mathrm{N}_{\mathrm{Lp}}$. The absorption bands at 1050 and $750 \mathrm{~nm}$ are attributed to the $S_{22}$ and $M_{11}$ transitions of the $1.5 \mathrm{~nm}$ diameter SWCNTs [36]. The Ag NP plasmon peak is present for most conditions, with positions ranging between 450 and $510 \mathrm{~nm}$. The blue shifted plasmon peaks of Ag NPs on SWCNTs seem to indicate that these NPs are significantly smaller than those on deposited on quartz. The high surface area of the SWCNTs film, as shown through SEM, provides a much higher effective density of nucleation sites onto which Ag can deposit and form NPs. This leads to a higher density of Ag NPs and thus decreases the number of $\mathrm{Ag}$ atoms available for each NP. In addition to the shift in position, the transmission spectra show that the $\mathrm{Ag}$ plasmon peaks are noticeably weaker in NHs than on quartz despite the higher NP density. Proximity-induced charge transfer between SWCNTs and Ag could deplete the number of electrons available for surface plasmon generation in the Ag NPs [34, 37]. However, there is no evidence of charge transfer in the positions of the 2D band in Raman spectra, where no trace of doping of the SWCNTs was found for $N_{\mathrm{Lp}}<1500$. It seems, therefore, that this quenching of the plasmon peak could be caused by the different medium refractive index. Indeed, the effective plasmon scattering cross section is strongly affected by the medium's refractive index and the plasmon peak intensity decreases for higher medium refractive index [38]. SWCNTs' refractive index can be approximated to be similar to that of graphite $(n=2.6)$ [39] for visible light, which is much higher than that of the quartz used $(n=1.46)$. This large difference in the medium refractive index could partially account for the observed decrease in intensity. The medium also has a direct effect on the plasmon peak position; a higher refractive index also red-shifts plasmon peaks. At first glance, this appears to be in contradiction with the observation that the NHs plasmon peak is blue shifted when Ag NPs are deposited on SWCNTs with respect to when they are deposited on quartz. However, a blue shift induced by sufficiently small particles can balance the red shift induced by the high refractive index of SWCNTs. Similar to what was observed for on-quartz Ag NPs, the plasmon peak disappears entirely at $N_{\mathrm{Lp}}>1500$ with percolation of the $\mathrm{Ag} \mathrm{NP}$ coating. Finally, no plasmon peak could be measured at low $N_{\mathrm{Lp}}$. This is attributed to a combination of a smaller density of NPs and of plasmon peak quenching.

Through UV-Vis spectroscopy, we are able to show that there is a plasmon peak in the visible light range, which is desirable for improvement of solar PV devices. The effect of the Ag plasmon can also be directly assessed through 


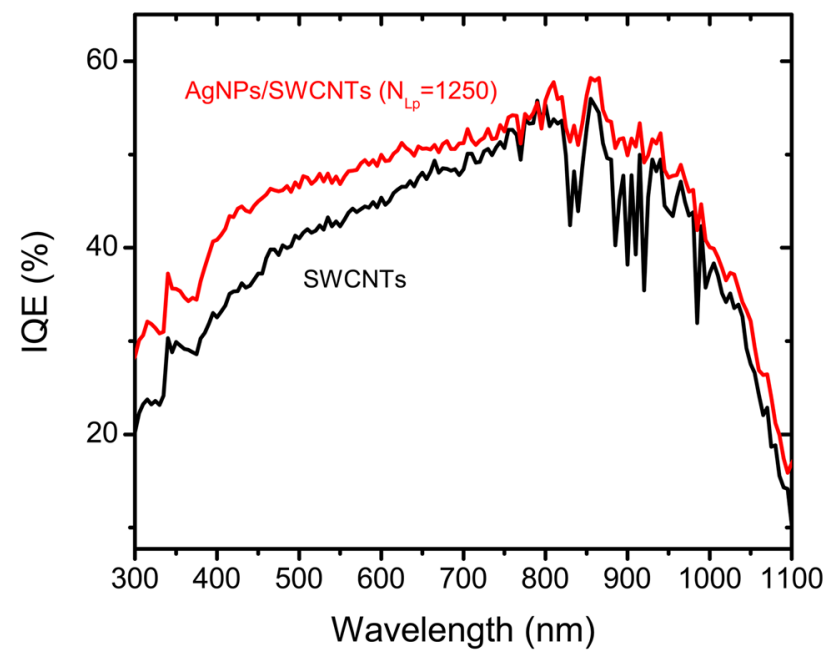

Fig. 4 Comparison of IQE spectra of SWCNTs/n-Si PV devices with and without Ag NPs

IQE measurements, in which a localized increase at the plasmon peak's wavelength would occur if Ag NPs contribute directly to photogeneration. On the other hand, if the Ag NPs strictly acted as an anti-reflection coating, the shape of IQE spectra of the SWCNTs/n-Si and AgNPsSWCNTs/n-Si devices would be very similar but shifted to higher efficiency values after decoration. Figure 4 compares the IQE spectra for the SWCNTs-Ag NPs n-Si devices with pristine SWCNTs devices. The IQE of the pristine device is about $20 \%$ in the UV range where it picks up and increases until the near infrared, where it declines towards $1100 \mathrm{~nm}$, close to the value of the bandgap of silicon $(1.11 \mathrm{eV})$. Overall, the IQE spectrum shape is similar to that of typical silicon-based devices, but with a somewhat lower IQE in the near UV and visible spectra [12]. This local decrease is attributed to the presence of the SWCNTs film that has a strong absorption peak in the UV (as shown in Fig. 3b). In comparison, the IQE spectrum of the Ag NPs-SWCNTs device starts at about $30 \%$ and is overall $10 \%$ higher until the IR range where it becomes equivalent to that of the pristine devices. The absence of a clearly defined plasmon-induced peak in the IQE spectra shows that the AgNPs act mostly as an antireflection layer, trapping a larger number of high energy photons close to the SWCNTs/n-Si interface.

\section{PCE optimization}

Preliminary IQE measurements have demonstrated that there is a net positive effect of the addition of Ag NPs to the SWCNTs films. Therefore, PCE was optimized as a function of $N_{\mathrm{Lp}}$. Figure 5a compares the $J-V$ curves for several values of $N_{\mathrm{Lp}}$ of NHs. The pristine SWCNTs films devices $\left(N_{\mathrm{Lp}}=0\right.$, red circles) show a high short-circuit
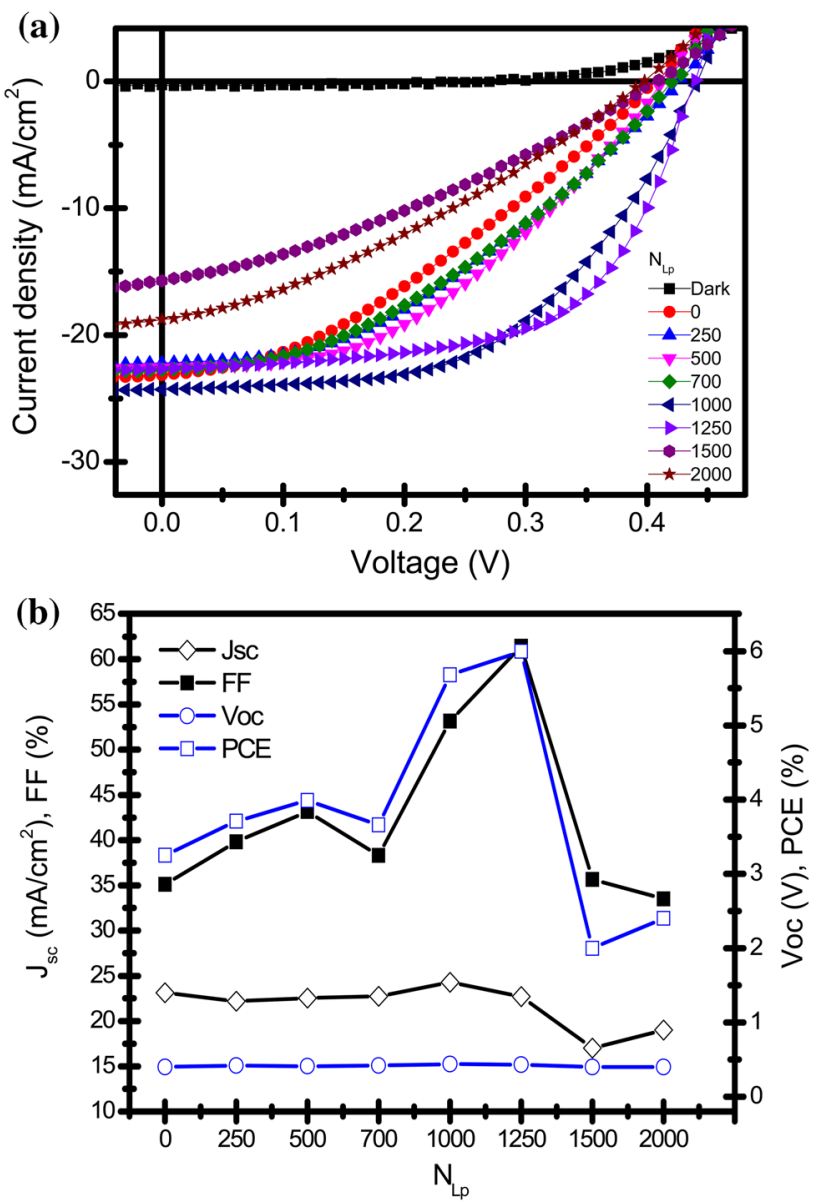

Fig. 5 a $J-V$ curves of PV devices at selected values of $N_{\mathrm{Lp}}$. b Comparison of the evolution of $J_{\mathrm{sc}}, \mathrm{FF}, V_{\mathrm{oc}}$ and the PCE as functions of $N_{\mathrm{Lp}}$

current $\left(J_{\mathrm{sc}}\right)$ value $\left(23 \mathrm{~mA} / \mathrm{cm}^{2}\right)$ and low fill factor values $(\mathrm{FF}=35 \%)$, which lead to a PCE of $3.5 \%$. Upon the addition of Ag NPs, PCE increases from a base value of $\sim 3.5 \%$ at $N_{\mathrm{Lp}}=0$, to $6 \%$ at $N_{\mathrm{Lp}}=1250$ as shown in Fig. $5 \mathrm{~b}$. Over the same range, $J_{\mathrm{sc}}$ also increases somewhat in value but stabilizes at $26 \mathrm{~mA} / \mathrm{cm}^{2}$. This variation in $J_{\mathrm{sc}}$ is too small to be the only driving force behind the increase in PCE. Open circuit voltage $\left(V_{\text {oc }}\right)$ has a somewhat low value $(0.37 \mathrm{~V})$ at $N_{\mathrm{Lp}}=0$ that is improved upon with the addition of Ag NPs up to $0.41 \mathrm{~V}$, in line with previously reported values [11]. FF follows a very similar pattern to that of PCE, increasing from $35 \%$ to $60 \%$ between $N_{\text {Lp }}=0$ and $N_{\text {Lp }}=1250$ (Fig. 5b). Both the PCE and FF decrease rapidly after the optimal point and the PCE plummets down to $2.5 \%$ for higher values of $N_{\mathrm{Lp}}$. Additionally, it can be seen that while $J_{\mathrm{sc}}$ decreases severely, $V_{\mathrm{oc}}$ remains in the same range.

These measurements clearly show that $N_{\mathrm{Lp}}=1250$ leads to an optimal value of PCE. There are several phenomena that can contribute to PCE optimization: doping and plasmonic effects, hot electron injection and electric 
effects through lowering of the overall film resistance. Doping as a means of increasing can effectively be ruled out. Indeed, as shown in the Raman spectra of Fig. 1, little to no doping appears until $N_{\mathrm{Lp}}=1500$ where undesirable n-type doping happens. While this could account for the decrease in PCE at high $N_{\mathrm{Lp}}$, it cannot be the underlying mechanism of the increase in PCE. Moreover, in addition to doping effects, plasmon contribution effects should be carefully considered. The IQE spectra of Fig. 4 show an increased intensity in the near UV that carries over into the visible range. This localized increase is attributed to the presence of Ag NPs and their plasmonic dispersion of light, which increases the chance of photons interacting with the SWCNTs/n-Si heterojunction. In effect, the integrated IQE (i.e., the area under the IQE curve) increases by about $12 \%$. Given that IQE and $J_{\mathrm{sc}}$ are directly correlated, it should be expected that this increase in EQE will similarly affect $J_{\mathrm{sc}}$. Indeed, $J_{\mathrm{sc}}$ is about $13 \%$ higher in SWCNTs/ Ag-NPs PV devices with respect to pristine SWCNTs PV devices. Finally, plasmon-enhanced hot electron injection possibly contributes to the increase in IQE and $J_{\text {sc }}$. In this model, the Ag NPs are considered to effectively act as a semiconductor from which sufficiently excited electrons could be injected directly in the SWCNTs films, rendering more charges available for collection. The efficiency of this process is known to be low and potentially counts for only a small fraction of the $J_{\mathrm{sc}}$ and IQE increase [40].

However, the increases in $J_{\mathrm{sc}}$ and IQE can only partially explain the more large increase in PCE. As shown in Fig. 5b, the main cause of the PCE improvement is the variation of the FF. The latter is directly related to the device properties (e.g., film resistance, contact resistances, etc.). The device's electric properties thus affect PCE more directly than it affects IQE or $V_{\mathrm{oc}}$. Figure 6 shows the electrical resistance of AgNPs/SWCNTs films. It can be

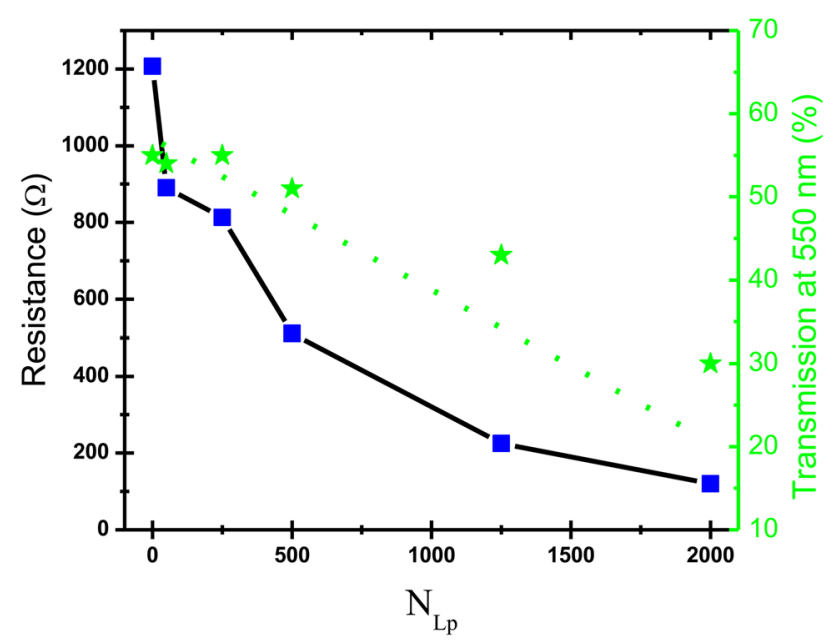

Fig. 6 Film resistance and optical transmission at $550 \mathrm{~nm}$ of SWCNTs/Ag-NPs hybrids as a function of $N_{\mathrm{Lp}}$ seen that the resistance decreases rapidly from 1200 to $200 \Omega$ over the whole range of $N_{\mathrm{Lp}}$, leading to the increase of the FF and thus of the PCE. However, partially counteracting this effect, optical transparency of the films decreases significantly from 60 to $30 \%$ over the same range of $N_{\mathrm{Lp}}$ as shown in Fig. 6. In addition to the decrease of the NHs resistance, there is a degradation in the ability of the $\mathrm{NHs} / \mathrm{n}-\mathrm{Si}$ junctions to act as a properly rectifying diode, thus contributing to the decrease key parameters such as FF and PCE. Indeed, the reverse bias current (i.e. the I-V curve's slopes at negative voltages) is more than five times smaller than the slopes of devices with low $\mathrm{N}_{\mathrm{Lp}}$ values. This also coincides with the switch from a discrete NPs Ag film to a continuous Ag NPs film as was evidenced through UV-Vis Spectroscopy and SEM. Also, Raman spectroscopy showed an n-type doping of the SWCNTs at $N_{\text {Lp }}>1250$, through the shifts of the G and 2D bands [38], which would also have in effect to degrade the rectification characteristics of the heterojunction with $\mathrm{n}$-Si. To summarize, the PCE SWCNTs/Ag NPs PV devices appear to be improved through a combination of $\mathrm{NH}$ film plasmonic effects and resistance lowering. These net positive effects are, however, eventually counterbalanced by Ag-induced doping of the SWCNTs that leads to poorer rectification and a decrease of the optical transparency of the Ag NP/ SWCNTs film.

\section{Conclusion}

In conclusion, we report in this paper a straightforward and clean way to decorate SWCNTs films with Ag NPs to form $\mathrm{NHs}$ that are directly integrated into PV devices. Using Raman spectroscopy, we can pinpoint the conditions at which the Ag NP film percolates and act more as a bulk silver film. We were also able to concomitantly identify this point in optical spectroscopy measurements and correlate this with PCE measurements. PCE of SWCNTs/n-Si devices is indeed increased from 3.5 to $6 \%$ through decoration of the SWCNTs films with Ag NPs. We show that the PV properties are directly related to the Ag NPs films' properties and to the various interactions between SWCNTs and Ag. Indeed, there is a combination of factors that contribute to the improvement of PCE. Raman spectroscopy measurements show that little to know the charge transfer occurs at low values of $N_{\mathrm{Lp}}$ but that major charge transfer-like phenomena hamper photogeneration at high $N_{\text {Lp }}$ values. However, through optical and electrical characterizations, we show that the PCE improvement appears to arise from a synergy between improved optical absorption and electrical conductivity. Future work should focus on numerical modeling of this device to better understand the importance of each factor. 
Open Access This article is distributed under the terms of the Creative Commons Attribution 4.0 International License (http:// creativecommons.org/licenses/by/4.0/), which permits unrestricted use, distribution, and reproduction in any medium, provided you give appropriate credit to the original author(s) and the source, provide a link to the Creative Commons license, and indicate if changes were made.

\section{References}

1. Dürkop, T., Getty, S.A., Cobas, E., Fuhrer, M.S.: Extraordinary mobility in semiconducting carbon nanotubes. Nano Lett. 4(1), 35-39 (2004)

2. Hecht, D.S., Hu, L., Irvin, G.: Emerging transparent electrodes based on thin films of carbon nanotubes, graphene, and metallic nanostructures. Adv. Mater. 23(13), 1482-1513 (2011)

3. Le Borgne, V., Gautier, L.A., El Khakani, M.A.: Figure of merit based maximization of the quantum efficiency of SWCNTs/n-Si photovoltaic devices. Appl. Phys. Lett. 103, 073103 (2013)

4. Ham, M.-H., Paulus, G.L.C., Lee, C.Y., Song, C., KalantarZadeh, K., Choi, W., Han, J.-H., Strano, M.S.: Evidence for highefficiency exciton dissociation at polymer/single-walled carbon nanotube interfaces in planar nano-heterojunction photovoltaics. ACS Nano 4(10), 6251-6259 (2010)

5. Park, J.-G., Akhtar, M.S., Li, Z.Y., Cho, D.-S., Lee, W., Yang, O.-B.: Application of single walled carbon nanotubes as counter electrode for dye sensitized solar cells. Electrochim. Acta 85, 600-604 (2012)

6. Ong, P.-L., Euler, W.B., Levitsky, I.A.: Hybrid solar cells based on single-walled carbon nanotubes/Si heterojunctions. Nanotechnology 21(10), 105203 (2010)

7. Jung, Y., Li, X., Rajan, N.K., Taylor, A.D., Reed, M.A.: Record high efficiency single-walled carbon nanotube/silicon $\mathrm{p}-\mathrm{n}$ junction solar cells. Nano Lett. 13(1), 95-99 (2013)

8. Kozawa, D., Hiraoka, K., Miyauchi, Y., Mouri, S., Matsuda, K.: Analysis of the photovoltaic properties of single-walled carbon nanotube/silicon heterojunction solar cells. Appl. Phys. Express 5(4), 42304 (2012)

9. Jia, Y., Li, P., Gui, X., Wei, J., Wang, K., Zhu, H., Wu, D., Zhang, L., Cao, A., Xu, Y.: Encapsulated carbon nanotube-oxidesilicon solar cells with stable $10 \%$ efficiency. Appl. Phys. Lett. 98(13), 133115 (2011)

10. Tune, D.D., Flavel, B.S., Krupke, R., Shapter, J.G.: Carbon nanotube-silicon solar cells. Adv. Energy Mater. 2(9), 1043-1055 (2012)

11. Tune, D.D., Blanch, A.J., Krupke, R., Flavel, B.S., Shapter, J.G.: Nanotube film metallicity and its effect on the performance of carbon nanotube-silicon solar cells. Phys. Status Solidi A 211(7), 1479-1487 (2014)

12. Le Borgne, V., Gautier, L.A., Castrucci, P., Del Gobbo, S., De Crescenzi, M., El Khakani, M.A.: Enhanced UV photoresponse of KrF-laser-synthesized single-wall carbon nanotubes/n-silicon hybrid photovoltaic devices. Nanotechnology 23(21), 215206 (2012)

13. Li, Z., Kunets, V.P., Saini, V., Xu, Y., Dervishi, E., Salamo, G.J., Biris, A.R., Biris, A.S.: Light-harvesting using high density p-type single wall carbon nanotube/n-type silicon heterojunctions. ACS Nano 3(6), 1407-1414 (2009)

14. Shi, E., Zhang, L., Li, Z., Li, P., Shang, Y., Jia, Y., Wei, J., Wang, K., Zhu, H., Wu, D., Zhang, S., Cao, A.: $\mathrm{TiO}_{2}$-coated carbon nanotube-silicon solar cells with efficiency of $15 \%$. Sci. Rep. 2 , 884 (2012)

15. Aïssa, B., El Khakani, M.A.: The channel length effect on the electrical performance of suspended-single-wall-carbon- nanotube-based field effect transistors. Nanotechnology 20(17), 175203 (2009)

16. Li, Z., Kunets, V.P., Saini, V., Xu, Y., Dervishi, E., Salamo, G.J., Biris, A.R., Biris, A.S.: $\mathrm{SOCl}_{2}$ enhanced photovoltaic conversion of single wall carbon nanotube/n-silicon heterojunctions. Appl. Phys. Lett. 93(24), 243117 (2008)

17. Bai, X., Wang, H.G., Wei, J.Q., Jia, Y., Zhu, H.W., Wang, K.L., $\mathrm{Wu}$, D.H.: Carbon nanotube-silicon hybrid solar cells with hydrogen peroxide doping. Chem. Phys. Lett. 533, 70-73 (2012)

18. Li, X., Jung, Y., Huang, J.-S., Goh, T., Taylor, A.D.: Device area scale-up and improvement of SWNT/Si solar cells using silver nanowires. Advanced Energy Materials 4(12), (2014)

19. Schaadt, D.M., Feng, B., Yu, E.T.: Enhanced semiconductor optical absorption via surface plasmon excitation in metal nanoparticles. Appl. Phys. Lett. 86, 063106 (2005)

20. Chen, X., Jia, B., Saha, J.K., Cai, B., Stokes, N., Qiao, Q., Wang, Y., Shi, Z., Gu, M.: Broadband enhancement in thin-film amorphous silicon solar cells enabled by nucleated silver nanoparticles. Nano Lett. 12(5), 2187-2192 (2012)

21. Chen, L., Zhang, S., Chang, L., Zeng, L., Yu, X., Zhao, J., Zhao, $\mathrm{S}$., $\mathrm{Xu}, \mathrm{C} .:$ Photovoltaic conversion enhancement of single wall carbon-Si heterojunction solar cell decorated with $\mathrm{Ag}$ nanoparticles. Electrochim. Acta 93, 293-300 (2013)

22. Gautier, L.-A., Le Borgne, V., Delegan, N., Pandiyan, R., El Khakani, M.A.: Field electron emission enhancement of graphenated MWCNTs emitters following their decoration with Au nanoparticles by a pulsed laser ablation process. Nanotechnology 26(4), 045706 (2015)

23. Ka, I., Le Borgne, V., Ma, D., El Khakani, M.A.: Pulsed laser ablation based direct synthesis of single-wall carbon nanotubes/ $\mathrm{PbS}$-quantum-dots nanohybrids exhibiting strong, spectrally wide and fast photoresponse. Adv. Mater. 24, 6289-6294 (2012)

24. Ka, I., Ma, D., El Khakani, M.A.: Tailoring the photoluminescence of PbS-nanoparticles layers deposited by means of the pulsed laser ablation technique. J. Nanopart. Res. 13(6), 2269-2274 (2011)

25. Moskovits, M.: Surface-enhanced Raman spectroscopy: a brief retrospective. J. Raman Spectrosc. 36(6-7), 485-496 (2005)

26. Rinzler, A.G., Liu, J., Dai, H., Nikolaev, P., Huffman, C.B., Rodriguez-Macías, F.J., Boul, P.J., Lu, A.H., Heymann, D., Colbert, D.T., Lee, R.S., Fischer, J.E., Rao, A.M., Eklund, P.C., Smalley, R.E.: Large-scale purification of single-wall carbon nanotubes: process, product, and characterization. Appl. Phys. A Mater. Sci. Process. 67(1), 29-37 (1998)

27. Kataura, H., Kumazawa, Y., Maniwa, Y., Umezu, I., Suzuki, S., Ohtsuka, Y., Achiba, Y.: Optical properties of single-wall carbon nanotubes. Synth. Met. 103(1-3), 2555-2558 (1999)

28. Bachilo, S.M., Strano, M.S., Kittrell, C., Hauge, R.H., Smalley, R.E., Weisman, R.B.: Structure-assigned optical spectra of single-walled carbon nanotubes. Science 298(5602), 2361-2366 (2002)

29. Valmalette, J.-C., Tan, Z., Abe, H., Ohara, S.: Raman scattering of linear chains of strongly coupled $\mathrm{Ag}$ nanoparticles on SWCNTs. Sci. Rep. 4, 5238 (2014)

30. Kneipp, K., Kneipp, H., Itzkan, I., Dasari, R.R., Feld, M.S.: Ultrasensitive chemical analysis by Raman spectroscopy. Chem. Rev. 99, 2957-2976 (1999)

31. Kharlamova, M.V., Niu, J.J.: Donor doping of single-walled carbon nanotubes by filling of channels with silver. J. Exp. Theor. Phys. 115(3), 485-491 (2012)

32. Niu, Z.Q., Fang, Y.: Surface-enhanced Raman scattering of single-walled carbon nanotubes on silver-coated and gold-coated filter paper. J. Colloid Interface Sci. 303(1), 224-228 (2006)

33. Tsang, J.C., Freitag, M., Perebeinos, V., Liu, J., Avouris, Ph: Doping and phonon renormalization in carbon nanotubes. Nat. Nanotechnol. 2(11), 725-730 (2007) 
34. Lin, Y., Watson, K.A., Fallbach, M.J., Ghose, S., Smith, J.G., Delozier, D.M., Cao, W., Crooks, R.E., Connell, J.W.: Rapid, solventless, bulk preparation of metal nanoparticle-decorated carbon nanotubes. ACS Nano 3(4), 871-884 (2009)

35. Maciel, I.O., Campos-Delgado, J., Cruz-Silva, E., Pimenta, M.A., Sumpter, B.G., Meunier, V., López-Urías, F., Muñoz-Sandoval, E., Terrones, H., Terrones, M., Jorio, A.: Synthesis, electronic structure, and Raman scattering of phosphorus-doped single-wall carbon nanotubes. Nano Lett. 9(6), 2267-2272 (2009)

36. Itkis, M.E., Perea, D.E., Niyogi, S., Rickard, S.M., Hamon, M.A., Hu, H., Zhao, B., Haddon, R.C.: Purity evaluation of asprepared single-walled carbon nanotube soot by use of solution-phase near-IR spectroscopy. Nano Lett. 3(3), 309-314 (2003)
37. Stapleton, A.J., Afre, R.A., Ellis, A.V., Shapter, J.G., Andersson, G.G., Quinton, J.S., Lewis, D.A.: Highly conductive interwoven carbon nanotube and silver nanowire transparent electrodes. Sci. Technol. Adv. Mater. 14(3), 035004 (2013)

38. Kelly, K.L., Coronado, E., Zhao, L.L., Schatz, G.C.: The optical properties of metal nanoparticles: the influence of size, shape, and dielectric environment. Journal of Physical Chemistry B 107(3), 668-677 (2003)

39. Djurisic, A.B., Li, H.: Optical properties of graphite. J. Appl. Phys. 85(10), 7404-7410 (1999)

40. Clavero, C.: Plasmon-induced hot-electron generation at nanoparticle/metal-oxide interfaces for photovoltaic and photocatalytic devices. Nat. Photonics 8(2), 95-103 (2014) 